\title{
Application of Computed Tomography in Structure Analyses of Metal Matrix Syntactic Foams
}

\author{
I. Kozma, I. Zsoldos, G. Dorogi, and S. Papp
}

\begin{abstract}
In order to create 3D models used in finite element analysis, a reconstruction of the structure of metal foam composites reinforced with ceramic spherical shells is necessary. A new algorithm has been developed for reconstruction. The input of the algorithm consists of CT layer images which were created at the Szechenyi Istvan University, Györ, Hungary. The CT images were analyzed by the new algorithm. The algorithm seeks the contours of the spherical shells by gradually increasing the radii, also in CT images. A suitably accurate 3D CAD model can be created with algorithm.
\end{abstract}

Index Terms-Foam reconstruction, digital image processing, $\mu \mathrm{CT}$, circle fitting.

\section{INTRODUCTION}

Metallic foams have a special class which also satisfies the definition of particle reinforced metal matrix composites. These are metal matrix syntactic foams. Metal matrix syntactic foams have numerous perspective applications as covers, hulls, castings, or in the automotive and electromechanical industry because of their high energy absorbing and damping capability. In these porous materials porosity is ensured by the incorporation of ceramic hollow microspheres [1]. The microspheres are commercially available and they contain mainly various ceramic oxides [2], [3].

The most important properties of metal matrix syntactic foams are compressive strength and absorbed energy [4]-[10].

The quality and chemical composition of the microspheres influence many properties of syntactic foams. They also have a strong influence during the production of the syntactic foams. The foams are usually produced by mixing techniques and gravitational casting or by pressure infiltration [11]-[13].

Because of the strong effect of the quality of the microspheres on the mechanical and other properties of the foams, the investigation of the structure of syntactic foams is very important. CT-based reconstruction methods can be applied to create real 3D models of the syntactic foams. CT-based reconstruction methods are used successfully in many different materials research fields such as the research of porous polymeric materials (foams) [14], and open celled and closed-cell metallic foams [15], [16]. Recently X-ray computed micro tomography has been applied on porous fuel

Manuscript received May 8, 2014; revised July 31, 2014. The research work presented in this paper was carried out as part of the TÁMOP-4.2.2.A-11/1/KONV-2012-0029 project in the framework of the New Széchenyi Plan. The realization of this project is supported by the European Union, and co-financed by the European Social Fund.

I. Kozma is with the Széchenyi István University, Hungary (e-mail: kozma@sze.hu). cell materials [17] and soil samples [18]. The history, the production, the geometrical, mechanical, thermal properties the dynamical behaviour and the fatigue processes of hollow spheres were studied in details [19]. Structural characterisation and morphology of metal foams were studied with models reconstructed from CT images [20].

\section{RECONSTRUCTION AlgORITHMS}

In this paper our goal was the $3 \mathrm{D}$ reconstruction of the structure of a specimen of $\mathrm{Al}$ composite reinforced with ceramic spherical shells. The starting point was the composite specimen (see Fig. 1). The dimensions of the cylindrical specimen are $\varnothing 15 \times 22.5 \mathrm{~mm}$. The basis for reconstruction was $\mathrm{CT}$ imaging. Fig. $1 \mathrm{~b}$ shows some tomograms of the specimen and one tomogram is detailed in Fig. 1c. The CT tomograms were made in the laboratory of the Materials Science Department of Széchenyi István University with a YXILON CT Modular industrial CT system, with a $225 \mathrm{kV}$ micro focus $\mathrm{X}$-ray tube and a resolution of $7 \mu \mathrm{m}$.

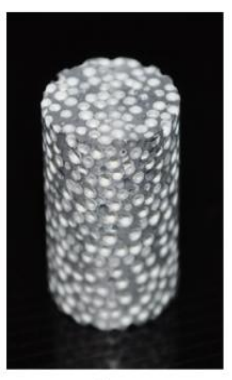

a

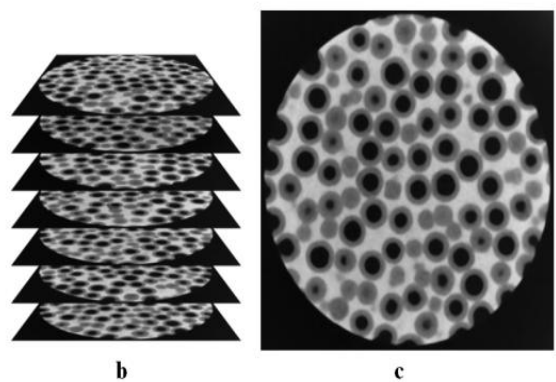

b
Fig. 1. a: The specimen of Al composite reinforced with ceramic spherical shells. b: A series of CT images. c: One CT image.

Software that processes CT images (e.g. Mimics, Geometry and the software that comes with the YXlon CT system) is able to reconstruct air bubbles detected in the structure (e.g. in castings) in the form of spheres. This reconstruction, however, only models inner surfaces in the case of ceramic spherical shells, outer surfaces cannot be reconstructed this way. In analysing CT images it was seen that the wall thickness of ceramic spherical shells is not uniform, and in addition, in some cases the outer and inner spherical surfaces are not concentric. To make reconstruction even more complicated, the structure also contains numerous intersecting spheres. Because of these problems, the full reconstruction of the structure could not be done directly with the available CT software. The models of the inner spherical surfaces of the ceramic spherical shells, however, could be created from the measured data, and these were used in building up the full model of the composite. 
We set out to reconstruct the outer spherical surfaces of the ceramic spherical shells by detecting the circles found in the CT images. To detect the circles we first used the Hough Circle algorithm [21]. Fig. 2a shows a ceramic spherical shell section selected from a CT image. It can be seen that the outer contour is not a perfect circle. The Hough Circle algorithm fits circles to several segments of the contour, to several arcs, as can be seen in Fig. 2b. In most cases, however, none of these circles is a circle best fitted to the actual outer contour. To determine the circles (or spheres in the 3D model) fitting the outer contours of the spherical shells well, we developed our own algorithms. We developed an algorithm, the radius incrementation algorithm.

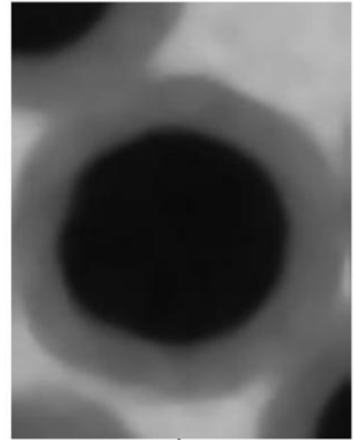

a

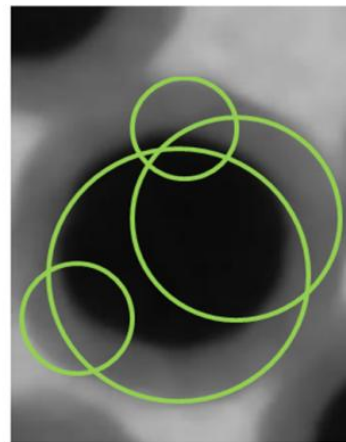

b
Fig. 2. a: Section of a CT image of a ceramic spherical shell. b: Some circles fitted to various circle sections with the Hough Circle algorithm.

\section{The ALGORITHM OF RADIUS INCREMENTATION}

The method of radius incrementation is based on the fact that the inner sphere of spherical shells is known. The algorithm consists of the following steps:

1) The starting point in a CT image is the inner, black disks.

2) The radius of the inner circles is incremented pixel by pixel.

3) After every step of incrementation it is examined whether the edge of the outer circle is reached. In order to do this, the proportion of light grey pixels representing the aluminium matrix to all the pixels intersected by the circle of incremented radius is calculated.

4) Experience shows that a circle is well fitted when the ratio of intersected grey pixels to all intersected pixels reaches $50 \%$.

5) If the ratio of intersected dark grey pixels increases, the algorithm is stopped immediately because this means intersecting circles with the neighbouring annulus being "penetrated".

The series of the above steps is also a simple, easily programmable task. A problem can arise when the inner and outer circles are not concentric. Therefore after each step of incrementation a correction of the centre is performed.

In the course of this correction the centre is moved in one step by one pixel towards the low intensity (dark grey pixels) points of the pixels intersected by the circle of increased radius. The shift of the centre is determined by the vector which is the resultant of all the vectors pointing from the centre to the low intensity pixels. After this control is given back to incrementation and the algorithm decides whether the radius or the centre needs to be changed further. In the ceramic spherical shell section shown in Fig. 3 the inner and outer circles are not concentric. For this reason the centre had to be moved a few pixels (purple pixels) in the course of the radius incrementation algorithm.

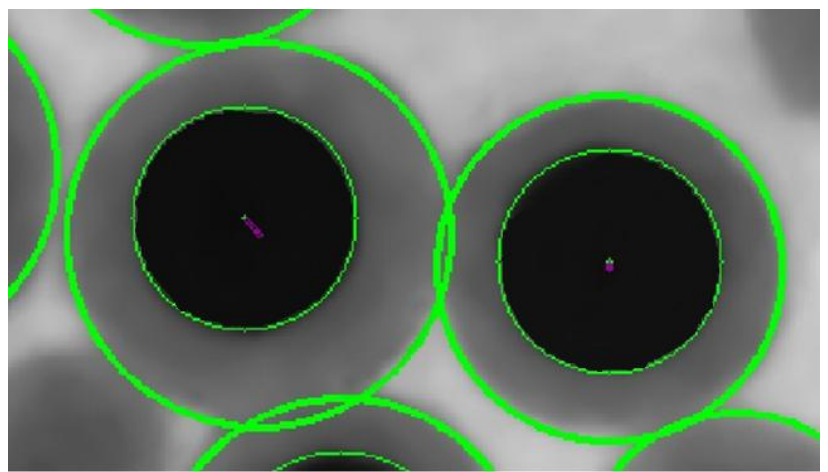

Fig. 3. Centre correction in the radius incrementation algorithm.

\section{Testing the Algorithms}

The algorithm are used to evaluate CT images of syntactic metal foam samples. The quality of the fitting of circles was determined by comparing the detected areas and the fitted circular areas.

In Fig. 4 the evaluation of two CT images is shown for thr algorithm. The slope of the linear trend line calculated for the points determined by the detected area and the area of the fitted circle is almost precisely " 1 " in the diagrams. The areas were defined with the number of pixels, the area of one pixel is $0.0009 \mathrm{~mm}^{2}$.
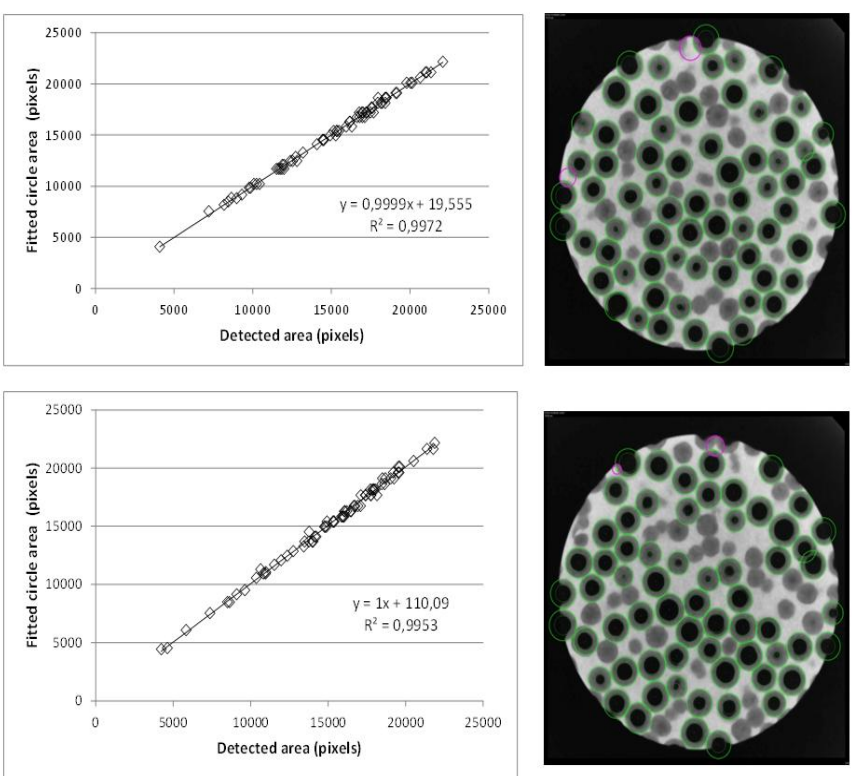

Fig. 4. Circles (in green) fitted with the radius incrementation algorithm in CT images. The diagrams show the points determined by the detected area and the fitted circle area, and the calculated linear trendline for the CT image on the right.

Theoretically the comparison of the detected areas and the fitted circle areas is not enough to evaluate the algorithms since it can happen that the areas of two shapes are identical yet the shapes are different. In Fig. 5a for example, a circle was fitted to a square of identical area. Fig. $5 \mathrm{~b}$ shows a circle fitted to a shape detected in a CT image.

Significant errors similar to errors of fitting a circle to a square cannot occur in the circularity algorithm. That 
algorithm can filter out such big errors using the average circularity parameter calculated from several rotations. The radius incrementation algorithm, however, theoretically does not exclude the possibility of significant errors.

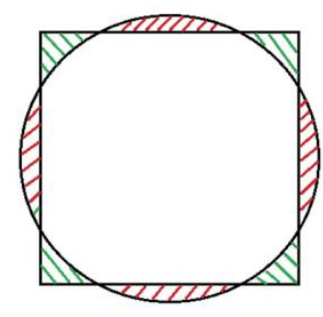

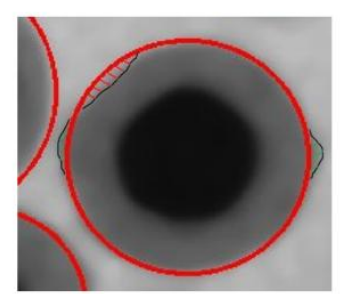

Fig. 5. Outer and inner errors in the case of circles fitted to various shapes. a: in the case of a circle fitted to a square, $b$ : in the case of a circle fitted to a shape in a CT image.

The errors of fitting the circles were determined using outer and inner errors of the circles, too. Inner and outer errors were defined as the following:

- Inner errors: the number of pixels found within the fitted circle but having the intensity of the material of the aluminium matrix (the areas shaded in red in Fig. 5).

- Outer errors: the number of pixels found outside the fitted circle but having the intensity of the material of the ceramic spherical shell which do not belong to any of the other detected areas (the latter condition is necessary because of the intersecting circles). These errors are shaded in green in Fig. 5 .

In the case of each detected circle the ratio of the sum of the inner and outer errors to the area of the fitted circle was calculated. This percentage was considered the error of a fitted circle even if this error does not only show the error of fitting but also the deviation of the shape of the detected area from the regular circular shape.

The above-mentioned errors were calculated for 100 analyzed CT images and their average and standard deviation were also calculated. The results can be found in Table I.

TABLE I: THE AVERAgE FITTING ERRORS OF CIRCLES WITH THE INTERPRETATION OF OUTER AND INNER ERRORS

\begin{tabular}{lll}
\hline \hline & $\begin{array}{l}\text { Average error of } \\
\text { fitting }\end{array}$ & $\begin{array}{l}\text { Standard } \\
\text { deviation }\end{array}$ \\
\hline Radius incrementation & $1,0-1,5 \%$ & $0,9-1,3 \%$
\end{tabular}

\section{Results AND THE CAD MODEL}

900 CT images were taken of the specimen in Fig. 1. The circle-detection algorithms were run on all images. The calculation of the radius of a sphere from two sections is shown in Fig. 6.

Using the notation in Fig. 6, the radius of the sphere $(r)$ can be calculated with the following formula:

$$
r=\sqrt{\frac{\left[(a-b)^{2}+h^{2}\right]\left[(a+b)^{2}+h^{2}\right]}{4 h^{2}}}
$$

where " $a$ " and " $b$ " are the radii of circular sections in two different CT images, and " $h$ " is the distance between the sections.

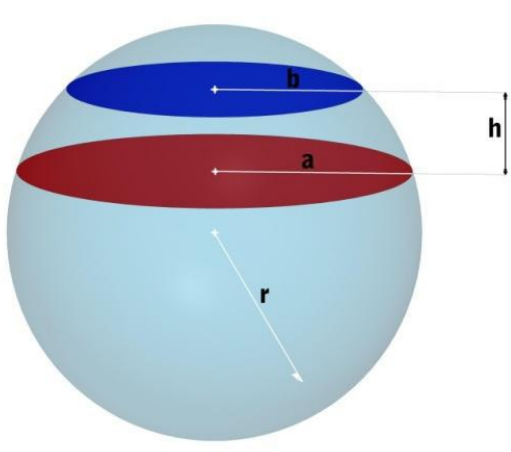

Fig. 6. Sphere parameters calculated from circular sections of CT images.

The formula may change if the two circular sections are in two separate hemispheres. When reconstructing a ceramic spherical shell, care was taken to use sections from the same hemisphere to calculate the radius of the sphere and the coordinates of the centre.

A CAD model was created from the data of the detected spheres, on which various engineering analyses (e.g. finite element analysis) can be run. Fig. 7 shows the CAD model.

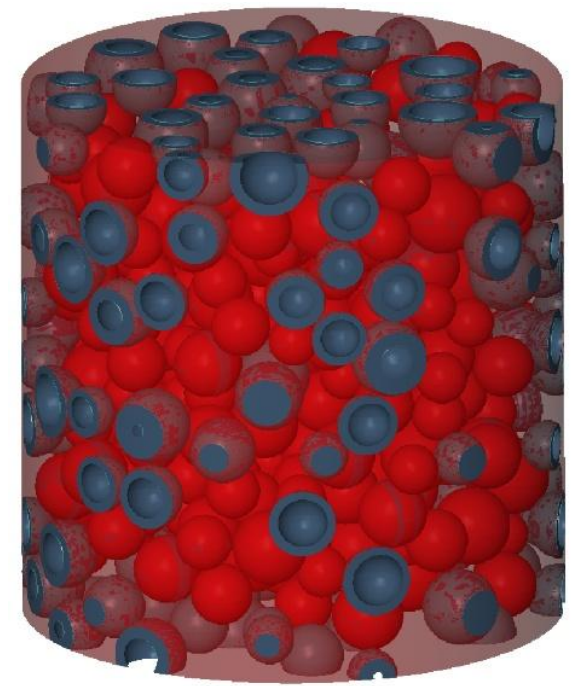

Fig. 7. The CAD model.

\section{SUMMARY}

The radius incrementation algorithm is developed and analysed for the CT-based reconstruction of aluminium composites reinforced with ceramic balls. The algorithms can well reconstruct the envelope circles of ceramic spherical shells found in CT images, even in the case of densely packed, contiguous or intersecting spherical shells. The reconstructed CAD model can be easily generated with the help of the new algorithm.

\section{REFERENCES}

[1] I. N. Orbulov and J. Dobránszky, "Producing metal matrix syntactic foams by pressure infiltration," Periodica Polytechnica Mechanical Engineering, vol. 52, pp. 35-42, 2008.

[2] Envirospheres Ltd. (2011). [Online]. Available: http://www.envirospheres.com/products.asp

[3] Sphere Services Inc. [Online]. Available: http://www.sphereservices.com/

[4] G. H. Wu, Z. Y. Dou, D. L. Sun, L. T. Jiang, B. S. Ding, and B. F. He, "Compression behaviors of cenosphere-pure aluminum syntactic foams," Scripta Materialia, vol. 56, pp. 221-224, 2007. 
[5] I. N. Orbulov and J. Ginsztler, "Compressive characteristics of metal matrix syntactic foams," Composites Part A, vol. 43, pp. 553-561, 2012.

[6] I. N. Orbulov and K. Májlinger, "Microstructural aspects of ceramic hollow microspheres reinforced metal matrix composites," International J. of Materials Research, vol. 104, pp. 903-911, 2013.

[7] P. K. Rohatgi, J. K. Kim, N. Gupta, S. Alaraj, and A. Daoud, "Compressivecharacteristics of A356/fly ash cenosphere composites synthesized by pressure infiltration technique," Composites Part A: Applied Science and Manufacturing, vol. 37, issue 3, pp. 430-437, March 2006.

[8] R. A. Palmer, K. Gao, T. M. Doan, L. Green, and G. Cavallaro, "Pressure infiltrated syntactic foams - Process development and mechanical properties," Materials Science and Engineering: A, vol. 464, issues 1-2, pp. 85-92, August 2007.

[9] D. K. Balch, J. G. O. Dwyer, G. R. Davis, C. M. Cady, G. T. Gray III, and D. C. Dunand, "Plasticity and damage in aluminum syntactic foams deformed under dynamic and quasi-static conditions," Materials Science and Engineering: A, vol. 391, issues 1-2, pp. 408-417, January 2005.

[10] W. J. Drury, S. A. Rickles, T. H. Sanders, and J. K. Cochran, "Light weight alloys for aerospace applications," The Minerals Metals and Materials Society, pp. 311-322, 1998.

[11] P. K. Rohatgi, R. Q. Guo, H. Iksan, E. J. Borchelt, and R. Asthana, "Pressure infiltration technique for synthesis of aluminum-fly ash particulate composite," Materials Science and Engineering A., vol. 244 pp. 22-30, 1998.

[12] T. Bárczy and G. Kaptay, "Modelling the infiltration of liquid metals into porous ceramics," Materials Science Forum, vol. 473-474, pp. 297-302, 2005

[13] P. K. Trumble, "Spontaneous in -filtration of non-cylindrical porosity: Close-packed spheres," Acta Materiala, vol. 46, pp. 2363-2367, 1998

[14] A. Léonard, C. Calberg, G. Kerckhofs, M. Wevers, R. Jérôme, J. P. Pirard, A. Germain, and S. Blacher, "Characterization of the porous structure of biodegradable scaffoldsobtained with supercritical CO2 as foaming agent," J. Porous Mat, vol. 15, pp. 397-403, 2008.

[15] J. Vicente, J. V. Daurelle, F. Topin, and L. Tadrist, "Open celled material structural properties measurement: From. Morphoplogy to transport properties," Materials Transactions, vol. 47, no. 9, pp. 2195-2202, 2006.

[16] I. Jeon, K. Kato, T. Sonod, T. Asahin, and K. J. Kang, "Cell wall mechanical properties of closed-cell Al foam," Mechanics of Materials vol. 41 , pp. $60-73,2009$.
[17] E. A. Wargo, T. Kotaka, Y. Tabuchi, and E. C. Kumbur, "Comparison of focused ion beam versus nano-scale X-ray computed tomography for resolving 3-D microstructures of porous fuel cell materials," Journal of Power Sources, vol. 241, pp. 608-618, 2013.

[18] A. N. Houston, S. Schmidt, A. M. Tarquis, W. Otten, P. C. Baveye, and S. M. Hapca, "Effect of scanning and image reconstruction settings in $\mathrm{X}$-ray computed tomography on soil image quality and segmentation performance," Geoderma, vol. 207-208, pp. 154-165, 2013.

[19] A. Oechsner and C. Augustin, Multifunctional Metallic Hollow Sphere Structures, Springer, 2009.

[20] M. Vesenjak, M. Borovinsek, and T. Fiedler, "Structural characterisation of advanced pore morphology (APM) foam elements," Materials Letters, vol. 110, pp. 201-203, 2013.

[21] H. K. Yuen, J. Princen, J. Illingworth, and J. Kittler, "Comparative study of hough transform methods for circle ending," Image Vision Comput., vol. 8, pp. 71-77, 1990.

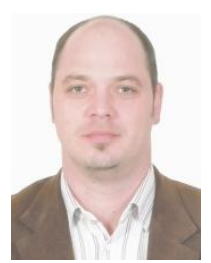

István Kozma was born in Győr on August 18, 1977. As a mechanical engineer, he got the BSc degree from Széchenyi István University, Győr, Hungary in 1999, then as a mechatronic engineer, he got the MSc degree from Széchenyi István University, Győr, Hungary in 2009.

He has great experience in surface digitalization and he has dealed with computed tomography for 4 years. He belongs to the staff of the Department of Materials Science and Technology of Széchenyi István University in Győr, and working there as an assistant lecturer. His main publications are: I. Kozma and E. Halbritter, "Measurement of the diameter of the imprint based on image processing using MathCAD and the evaluation software of an industrial CT," Acto Technica Jaurinensis, vol. 6. No. 2, pp. 48-58, 2013; I. Czinege, F. Csizmazia, and I. Kozma, "New opportunities in non-destructive testing (in Hungarian) The Future's Vehicle," A JÖVÖ JARMÜVE : JÁRMÜIPARI INNOVÁCIÓ, vol. 7, pp. 1-2, pp. 75-85, 2011; I. Kozma, F. Csizmazia, and I. Czinege, "Analysis of the geometrical precision of transmission and reflective methods for surface reconstruction (in Hungarian)," in Proc. Conference Proceedings of OGET, 2012, pp. 124-128.

Mr. Kozma is a member of the Society of Hungarian Material Testing and the Széchenyi Scientific Committee. 